\title{
Live-Cell Imaging of Transcriptional Activity at DNA Double-Strand Breaks
}

\author{
Madalena R. de Almeida ${ }^{1}$, Eduardo Gameiro ${ }^{1}$, Sérgio F. de Almeida ${ }^{1}$, Robert M. Martin ${ }^{1}$ \\ ${ }^{1}$ Instituto de Medicina Molecular João Lobo Antunes, Faculdade de Medicina da Universidade de Lisboa
}

\section{Corresponding Authors}

Sérgio F. de Almeida

sergioalmeida@medicina.ulisboa.pt

Robert M. Martin

robertmartin@medicina.ulisboa.pt

\section{Citation}

de Almeida, M.R., Gameiro, E., de Almeida, S.F., Martin, R.M. Live-Cell Imaging of Transcriptional Activity at DNA Double-Strand Breaks. J. Vis. Exp. (175), e62968, doi:10.3791/62968 (2021).

\section{Date Published}

September 20, 2021

\section{DOI}

$10.3791 / 62968$

URL

jove.com/video/62968

\section{Abstract}

DNA double-strand breaks (DSB) are the most severe type of DNA damage. Despite the catastrophic consequences on genome integrity, it remains so far elusive how DSBs affect transcription. A reason for this was the lack of suitable tools to simultaneously monitor transcription and the induction of a genic DSB with sufficient temporal and spatial resolution. This work describes a set of new reporters that directly visualize transcription in live cells immediately after the induction of a DSB in the DNA template. Bacteriophage RNA stem-loops are employed to monitor the transcription with single-molecule sensitivity. For targetting the DSB to a specific gene region, the reporter genes are engineered to contain a single recognition sequence of the homing endonuclease I-Scel, otherwise absent from the human genome. A single copy of each reporter gene was integrated into the genome of human cell lines. This experimental system allows the detection of single RNA molecules generated by the canonical gene transcription or by DNA break-induced transcription initiation. These reporters provide an unprecedented opportunity for interpreting the reciprocal interactions between transcription and DNA damage and to disclose hitherto unappreciated aspects of DNA break-induced transcription.

\section{Introduction}

DNA double-strand breaks (DSBs) are toxic DNA lesions that disrupt cell function and contribute to the insurgence of several diseases and ageing ${ }^{1}$. Mutations resulting from the inaccurate repair of DSBs impact gene expression and set the basis for the functional decline of the cell. The emergent view that DSBs drive de novo break-induced transcription at the lesion site $2,3,4,5,6,7$ suggests that DSBs may also affect cellular function through break- induced RNAs. Several recent studies indicate that DSBs are sufficient to initiate programmed (e.g., at stimulusinducible genes) and unscheduled (e.g., at non-canonical promoters) transcription $4,5,7$. However, despite several studies exploring the links between DNA damage and transcription, the field still lagged in its capacity to deliver a precise (i.e., single-molecule) characterization of the transcriptional events at DNA break sites. One 
important reason for this was the lack of appropriate experimental tools. Cell irradiation ( $\mathrm{\gamma}$-rays, X-rays, heavy ions) and drug treatments (e.g., topoisomerase inhibitors or intercalating agents) lack spatial precision and induce DNA lesions other than DSBs, including single-strand breaks and DNA adducts ${ }^{8}$. Endonucleases, such as I-Ppol and AsiSI, generate locus-specific DSBs but have not been combined with a system that allows simultaneous livecell visualization of transcription at a single locus with high temporal precision ${ }^{8}$. To bypass this limitation, our lab spearheaded developing a set of cutting-edge reporters that directly visualize transcription with single-molecule resolution upon the controlled induction of a unique $\mathrm{DSB}^{4}$. Here, we describe these reporters, provide a detailed protocol for livecell imaging of transcription at DSBs and show data revealing transcription initiation at a single DSB.

The reporter gene systems used in this protocol are based on the well-characterized mouse $\lg M$ reporter gene and contain the exons $\mathrm{M} 1$ and M2 of the membrane-bound form $(\mu \mathrm{m})$ of the $\operatorname{lgM} \mu$ heavy chain ${ }^{9,10,11}$. A hybrid intron separates the two exons with the strong adenovirus major late transcript (AdML) PY tract ${ }^{12}$. The expression of the reporter genes is controlled by the human cytomegalovirus promoter (CMV), into which two tandem copies of the Tet operator (TetO) sequence have been inserted. The reporter genes are each inserted into a plasmid vector containing an Flp Recombination Target (FRT) site and inserted into a specific FRT target site in the genome of a HEK293 host cell line. This cell line also constitutively expresses the Tet repressor protein to regulate the expression of the reporter gene via the presence or absence of tetracycline/doxycycline. To allow visualization of the reporter gene transcription, 24 tandem repeats of the MS2 stem loop sequence and 24 tandem repeats of the PP7 stem loop sequence were inserted at different positions in respect to transcription start site and exon/intron structure of the reporter gene. The MS2/PP7 RNA stem loops form upon transcription and are specifically bound by ectopically expressed MS2/PP7 coat proteins tagged with green and red fluorescent proteins, a strategy widely used before to image transcription $13,14,15$. In addition, a single copy of the $18 \mathrm{bp}$ recognition sequence was inserted for the homing endonuclease I-Scel that is directly flanked by the RNA stem-loop sequence arrays in the reporter genes. Standard cloning techniques were used to generate all plasmids, the fragment containing the I-Scel-24xMS2 stemloop of the PROP reporter gene was synthesized by a commercial gene synthesizing service.

The promoter-proximal DSB reporter gene (PROP) was constructed by inserting the I-Scel cutting site 45 base pairs (bp) downstream of the putative transcription start site in exon I, followed by 149 bp until the start of the $24 x$ MS2 stem-loop cassette, which was de novo designed with two alternating non-identical stem-loop sequences ${ }^{16}$ and additional five non-repetitive 20 bp spacer sequences to reduce redundancy. The MS2 stem-loop array is followed by $72 \mathrm{bp}$ until the beginning of the $1844 \mathrm{bp}$ intron and the 1085 bp exon II until the cleavage and polyadenylation site. The exon II encodes a cyan fluorescent protein (CFP) fused to a C-terminal peroxisomal targeting sequence (PTS) from the human peroxisomal acyl CoA oxidase to allow an independent screening of the reporter gene expression (Figure 1A).

The exon II DSB reporter gene (EX2) consists of a 167 bp exon I followed by the intron and exon II encoding the CFPPTS. Further downstream at a distance of $169 \mathrm{bp}$, a cassette containing 24x MS2 stem-loops was inserted, followed by an 84 bp linker sequence with an I-Scel site in the center, 
followed by 24x PP7 stem-loops and 221 bp until the cleavage and polyadenylation site ${ }^{17}$ (Figure 1B).

Lastly, the exon II DSB reporter gene with antisense transcription labeling (EX2AS) is based on the human ubiquitin B (UBB) gene transcript UBB-201 and contains two exons and one intron. The exon I have a total length of 1534 bp with an inverse insertion of the 24x MS2 stemloop sequence. Therefore, the correct MS2 stem-loop RNA sequence will be transcribed in an antisense direction with respect to the sense transcription of the reporter gene from the CMV promoter. The intron has a length of $490 \mathrm{bp}$, followed by exon II with the I-Scel site, and a coding region was inserted for two in-frame ubiquitin subunits. Downstream of the UBB gene is a sequence that forms a $24 x$ PP7 stem-loop upon transcription of the reporter gene in a sense direction (Figure 1C).

The transient transfection of an inducible construct of the homing endonuclease I-Scel allows for the controlled creation of a DSB at the inserted recognition site within each reporter gene ${ }^{18}$. The $l-S c e l$ endonuclease is fused in frame with the ligand-binding domain of the glucocorticoid receptor and a farred fluorescent protein iRFP713. This construct is cytoplasmic in the absence of triamcinolone acetonide (TA) but migrates rapidly into the nucleus upon the addition of TA to the growth medium of the cells (Figure 1D). The induction of DSBs by the I-Scel system is robust, as demonstrated before ${ }^{18,19,20}$. The reporter gene transcription can be monitored in parallel by visualizing the fluorescently tagged RNA stem-loop systems MS2 and PP7.

\section{Protocol}

\section{Preparation and transfection of cells for live-cell microscopy}

1. Prepare a $25 \mathrm{~cm}^{2}$ cell culture flask of the reporter cell line (EX2, EX2-AS, or PROM) with $5 \mathrm{~mL}$ of DMEM to achieve $80-90 \%$ confluency on the day before the livecell microscopy experiment.

2. Aspirate the medium with a pipette from the $25 \mathrm{~cm}^{2}$ cell culture flask and wash the cells with $2.5 \mathrm{~mL}$ of $1 \times$ PBS.

3. Add $1 \mathrm{~mL}$ of trypsin-EDTA $(0.05 \%)$ and incubate at $37^{\circ} \mathrm{C}$ for 2-3 min for cell detachment.

4. After cell detachment, add $4 \mathrm{~mL}$ of DMEM without phenol red containing HEPES buffer, supplemented with $10 \%$ (v/v) charcoal-stripped fetal bovine serum, and gently resuspend the cells.

5. Plate $1 \mathrm{~mL}$ of the cell solution in a $35 \mathrm{~mm}$ round dish with $10 \mathrm{~mm}$ glass-bottom well (diameter No. 1.5) and homogenize. Store the $35 \mathrm{~mm}$ round dish inside a 100 $\mathrm{mm}$ standard cell culture dish and incubate it at $37^{\circ} \mathrm{C}$ in a humidified atmosphere with $5 \% \mathrm{CO}_{2}$.

6. $\sim 6 \mathrm{~h}$ after seeding, transfect the cells in the glass bottom dish. For each transfection mixture, prepare two solutions in the following manner:

1. In a $1.5 \mathrm{~mL}$ microcentrifuge tube, prepare solution A containing $150 \mu \mathrm{L}$ of reduced-serum minimal essential medium (MEM), plasmid DNA (as described in Table 1), and $2.5 \mu \mathrm{g} / \mu \mathrm{L}$ of DNA of transfection helper reagent (see Table of Materials). 
2. In parallel, prepare solution $\mathrm{B}$, containing $150 \mu \mathrm{L}$ of reduced-serum MEM and $1.5 \mu \mathrm{g} / \mu \mathrm{L}$ DNA of a lipid-based transfection reagent (see Table of Materials).

3. Incubate both solutions at room temperature (RT) for 5 min. Then, gently add solution A to solution B and incubate $20 \mathrm{~min}$ at RT.

4. To transfect the cells, add $300 \mu \mathrm{L}$ of solution $\mathrm{A}+\mathrm{B}$ dropwise to each dish and gently distribute. Store the glass bottom dish inside a $100 \mathrm{~mm}$ standard cell culture dish and incubate it at $37^{\circ} \mathrm{C}$ in a humidified atmosphere with $5 \% \mathrm{CO}_{2}$.

7. Prepare a $1.5 \mathrm{~mL}$ microcentrifuge tube with $200 \mu \mathrm{L}$ of DMEM with HEPES, without phenol red supplemented with $10 \%(\mathrm{v} / \mathrm{v})$ charcoal-stripped fetal bovine serum and add TA to a concentration of $7.5 \times 10^{-7} \mathrm{M}$.

\section{Experimental setup}

1. Set the temperature of the large plexiglass microscope incubation chamber and the top stage incubation chamber to $37{ }^{\circ} \mathrm{C}$ at the common control unit. Set the environmental conditions inside the stage incubation chamber to $5 \% \mathrm{CO}_{2}$ and $100 \%$ humidity.

NOTE: The microscope cage and stage incubator temperature must be set up at least $1 \mathrm{~h}$ before starting the experiment to allow heating of the complete system to minimize temperature fluctuations. Start all other microscope control and operating units at the same time.

2. Induce the transcription of the reporter genes by adding doxycycline $(0.5 \mu \mathrm{g} / \mathrm{mL})$ to the growth medium and gently mix by pipetting up and down with a $200 \mu \mathrm{L}$ micropipette $\sim 1 \mathrm{~h}$ before starting the microscopy observation.
NOTE: Keep the glass-bottom dish with the transfected cells is inside a $100 \mathrm{~mm}$ standard cell culture dish for easy handling and transport from the cell culture to the microscope room in a styrofoam container to maintain the temperature as stable as possible.

3. Transport the cells to the microscope at least $30 \mathrm{~min}$ before starting the observation and upon arrival, place the $100 \mathrm{~mm}$ dish with the cells immediately inside the preheated large microscope incubation chamber.

4. Place the microcentrifuge tube with the pre-diluted TA from step 1.7. inside the large microscope environmental chamber to warm up to $37^{\circ} \mathrm{C}$.

5. Replace the lid of the glass bottom dish similar to that prepared before with a $3 \mathrm{~mm}$ diameter hole drilled into the lid (Figure 2A).

NOTE: The TA will be added later to the cells through the small hole in the lid without manipulating the dish mounted on the microscope stage.

6. Select the 100x (apochromatic objective, 1.4 numerical aperture) oil immersion objective in the microscope control panel. Apply a drop of immersion oil to the objective.

1. Set the glass-bottom dish with the cells inside the microscope stage incubation chamber and lock it in place. Close the lid of the stage incubator and all doors of the microscope housing.

7. Start the microscope operating and control software, open the Focus Control window (Figure 2B), click on the Scope pane, and in the Emission Selection pane, click the $100 \%$ Eye box to set the ocular beam path for direct sample observation by eye. 
1. In the Filter Set menu, switch to Eye filter set and click Brightfield, and press the Open Brightfield button.

8. Move the microscope objective towards the glass bottom dish until the oil touches the glass. Then look through the oculars and manually focus on the plane of the cells. Switch off the Open Brightfield button.

9. Leave the cells for $30 \mathrm{~min}$ before starting the experimental observations to adapt to environmental conditions and prevent focal drift during imaging by temperature gradients.

10. Place a $200 \mu \mathrm{L}$ micropipette and $200 \mu \mathrm{L}$ filter tips ready to use aside at room temperature.

\section{Image acquisition}

1. In the Focus Control window of the microscope control software, set the laser intensity to $5 \%$ and enter a value of $50 \mathrm{~ms}$ for the exposure time (Figure 2B).

2. Open the Capture window to adjust the settings to perform an automated image acquisition of threedimensional (3D) time-lapses (Figure 2C).

3. Select the 3D capture acquisition type and set 12 to 16 optical slices separated by $0.4 \mu \mathrm{m}$, tick the checkboxes of Range around current and Return to current position after capture.

4. In the Timelapse Capture pane, enter a value of 120 for the \# of time points and $30 \mathrm{~s}$ for the interval.

5. Select the confocal filter set according to the transfected fluorescent protein labels with $\boldsymbol{\lambda}=\mathbf{4 8 8} \mathbf{n m}$ for GFP, $\lambda=$ $561 \mathrm{~nm}$ for TagRFPt, and $\lambda=640 \mathrm{~nm}$ for iRFP713 and set the exposure time for each channel to $\mathbf{5 0} \mathbf{~ m s}$.
6. Use the setting Current for the Laser power to use the value of $5 \%$ selected in the Focus Window (Figure 2C).

7. In the Focus Control window, go to the Camera pane, select the Scale image display control and choose the Manual button to set up a fixed range of image intensities to be displayed. Enter values for Low: $\mathbf{5 0 0}$ and High: 5000 (see Figure 2B).

NOTE: This setting limits the dynamic range of the camera capture displayed in the live view to select cells within the same range of fluorescence intensities (Figure 2D).

8. Select the cells for the 3D time-lapse imaging of transcription sites upon induction of a DSB. Screen the cells and select three fields of view according to the conditions described in the Discussion.

9. Focus each selected cell previously located in the center of the field of view with the transcription site in the middle plane of the Z-stack.

NOTE: Centering the cell and the transcription site in $X Y Z$ accommodates for some movement of the cell.

10. Mark each $X Y Z$ position in the $X Y$ pane of the Focus Control window by clicking Set Point.

NOTE: Re-visit the selected positions 2-3 times over the following $5 \mathrm{~min}$ to confirm continuous transcription of the reporter genes and relative positional stability of the positions of the cells in $X Y Z$ dimensions.

11. Add $200 \mu \mathrm{L}$ of the pre-diluted TA from step 1.7. to the cells as shown in Figure 2F.

NOTE: Take extreme care not to touch the glass-bottom dish or the lid while adding the TA to prevent any shift from the marked $X Y$ positions. After DSB induction, confirm that the cells are centered within the field of view, and the transcription site is in the center Z-plane. The re- 
focusing and position update should take not more than 1-2 min.

12. Start the 3D time-series imaging by clicking on Start on the Capture window.

13. Save the imaging data in the microscope control software data format on the microscope control computer hard drive.

\section{Data analysis}

1. Open the imaging data in the microscope control software and export as 16-bit TIFF-format files.

2. Open the exported files with the StaQtool ${ }^{21}$ software. Select the Single Spot 3D mode and load the image files by pressing Go.

NOTE: The selected time series opens in the Max Projection Timelapse Viewer, showing a maximum intensity projection of the z-stack of the first time point (Figure 3A).

3. Adjust the image intensity display with the vertical MAX slider on the left side.

4. Select the timepoint to analyze with the horizontal Timepoint slider.

5. Hover the cursor to the position of the labeled transcription site for manually marking or use the Auto detect function and press on the respective transcription site if several objects were detected.

6. Use the Auto Track function to determine the $X Y Z$ positions of the transcription site over time.

NOTE: If a given position was not tracked correctly, select the transcription site manually according to the StaQtool software manual.
7. Press the Auto button to perform the Gauss fitting for each timepoint and measure the total fluorescence intensity (TFI).

NOTE: If the transcriptional activity ceases, the tracking tool remains on the last position where a diffractionlimited object (a labeled transcription site) was detected. If the cell moves in $\mathrm{XY}$ direction after the transcription site label disappears, a manual repositioning of the search square is required.

8. After finishing the TFI value fitting for each timepoint, press the End timelapse button to close the current timeseries image file and continue to the next file.

NOTE: The TFI values are automatically exported and saved in an Excel file.

\section{Microscopy calibration measurements and analysis}

1. Seed cells into $35 \mathrm{~mm}$ glass-bottom dishes and transfect with the fluorescently tagged MS2 and PP7 coat proteins as described in Section 1.

NOTE: For the calibration measurements, use the same reporter gene cell lines described in the Introduction.

2. Add $0.5 \mu \mathrm{g} / \mathrm{mL}$ of doxycycline to the growth medium of the cells $1 \mathrm{~h}$ before starting the microscopy image acquisition.

3. Mount the glass bottom dish inside the microscope stage incubation chamber and prepare the image acquisition as before (see Section 2).

NOTE: The original lid of the glass bottom dish is not replaced for the calibration experiments.

4. Use the same Laser intensity and exposure settings as described in point 3.1. 
5. Set the Capture settings for 2D time-series (deselect the 3D option in the Capture Type pane) and set 120 timepoints at intervals of $500 \mathrm{~ms}$ in the Timelapse Capture panel (Figure 2C).

NOTE: This image acquisition setting will result in time series within a single optical plane at very short intervals.

6. Acquire dozens of calibration time series from multiple positions to generate data sets to count several hundred single transcripts TFI measurements.

7. For the analysis of the calibration time series, convert the files to 16-bit TIFF-format files accordingly to point 4.1.

8. Open the exported files with the StaQtool ${ }^{21}$ software (Figure 3). Press the Select LOG file button, choose the Logfile of the respective 2D time series acquired as described in point 4.2 .

9. Select the Multiple Spots 2D check box and press the $\mathbf{G O}$ button to load the time series into the analysis software.

10. In the Timelapse viewer window (Figure $3 A$ ), in the PSF FWHM, the input field inserts the value calculated for the microscope system and objective as described ${ }^{21}$.

11. To start the analysis process, press the Auto Detect button to detect all diffraction-limited objects for the current time point displayed. Then, click on AutoFit to perform the Gaussian Fitting to determine the TFI value for each object (Figure 3A).

12. Alternatively, point the cursor over a diffraction-limited object and click to select it (green circle within a white square appears), and press the Gaussian Fit button for the manual selection and Gauss fitting procedure.

NOTE: The last mode is recommended to exclude objects not counted, such as bright transcription sites with several nascent transcripts present in the same nucleus.

13. Press the End timelapse button to finish the previous step.

NOTE: The results are automatically saved in a Microsoft Excel file format in the same folder as the image file.

14. Start the TFI and W Distributions module for multiple spots by pressing the respective button (Figure 3B).

15. Load Excel files via the Add file button and start the TFI analysis by pressing the Go button.

NOTE: The output is the mean TFI value determined from multiple measured single transcripts TFIs.

16. Start the $\mathrm{W}$ analysis by inserting the PSF FWHM previously used in point 5.10. and press the Go button using the default value for the Bin.

NOTE: The W parameter is quality control for the single transcript TFI measurements to comply with the correct PSF FWHM value for the microscope system used.

\section{Data and calibration merging}

1. Enter the time series TFI values obtained from the Excel sheet saved in point 4.8. into a new Excel sheet and divide each time point value by the mean TFI for single transcripts obtained in point 5.15 .

NOTE: For standardizing this process, custom-prepared Excel template forms were used.

\section{Representative Results}

The cell lines harboring the reporter genes described in Figure 1A-C allow the study of transcription dynamics at a single DSB in live cells. The numbers in Figure 1A-C below each graphical reporter gene representation indicate the length in kilobase pairs (kbp). CMV indicates 
the cytomegalovirus promoter, TetO is the Tet-operator sequences, pA highlights the $3^{\prime}$ cleavage and polyadenylation site at the gene end. CFP-PTS is the encoded cyan fluorescent protein fused to a peroxisomal targeting signal, and $2 \mathrm{UBB}$ is the encoded human ubiquitin $B$ tandem unit. By following the protocol and analysis procedures described above, it is possible to obtain graphs displaying the number of fluorescently labeled reporter gene transcripts over time, with a temporal resolution of seconds over periods of up to hours (Figure 4A-D). The graph in Figure 4A displays the time course of TFI values of a PROM reporter gene transcription site labeled by the accumulation of MS2-GFP molecules on nascent transcripts. This particular graph represents a control experiment without TA addition; therefore, no DSB is induced. Transcription continues with burst-like peaks and 2-8 transcripts at a time over the entire observation period of $60 \mathrm{~min}$. Similar results were obtained for the EX2 and EX2-AS reporter genes (data not shown here) ${ }^{4}$. The induction of a single DSBs in the reporter genes (using I-Scel-GR-iRFP) allows studying the impact of the DSB on the ongoing reporter gene transcription and the monitoring of transcription events emerging from the DSB site, namely break-induced transcription (Figure 4B-D).

\section{The dynamics of DNA break-induced transcription depend on the location of the DSB within the gene}

The induction of a single DSB by I-Scel-GR-iRFP in the reporter gene with a promoter-proximal I-Sce/ recognition site leads to transcriptional silencing of the reporter gene after around $11 \mathrm{~min}$ after TA addition, and the transcription is not restored within the 60 min observation period (Figure 4B). When observing the EX2 reporter gene transcription, complete suppression of the canonical promoter-driven transcription was detected by a simultaneously complete loss of both MS2-GFP and PP7-RFP signals around 30 min after TA addition. However, within 10 min, transcription restarts, as revealed by re-appearing peaks of PP7-RFP fluorescence. The complete recovery of the PP7-RFP signal (and not the MS2-GFP fluorescence) shows break-induced transcription initiation (Figure 4C). The break-induced transcription is not stable over long periods; it appears burst-like and low peak intensity, indicating that only a few break-induced transcripts were initiated from the DSB site.

The EX2AS reporter gene, which contains a 24x PP7 stemloop array in exon II downstream of the I-Scel site to detect the sense transcription, shows the canonical promoter-driven transcription terminating within EX2AS reporter gene within approximately $25 \mathrm{~min}$ after TA addition. It is then replaced by antisense break-induced transcription, as revealed by the accumulation of MS2-GFP protein binding to RNA generated from antisense MS2 stem-loop sequences (Figure 4D). The antisense transcriptional activity was absent before the interruption of the sense transcription due to the induction of the DSB and shows in this example that several transcripts were initiated from the break site within around $15 \mathrm{~min}$. The representative data obtained here show that DSBs have different effects on transcription depending on their location within the gene, as was recently reported ${ }^{4}$. The data also reveal a cell to cell variability in the timing of the DSB induction by I-Scel-GR-iRFP, which ranges from 12 to 30 min after TA addition. Furthermore, the detection of individual transcripts allows the discovery of cell to cell and break site differences towards the intensity of the break-induced transcriptional activity. The break-induced transcription is only detected at DSBs within the gene. It is absent at promoter-proximal DSBs, where the canonical transcription ceases upon a DSB for the remaining observation period. 
A

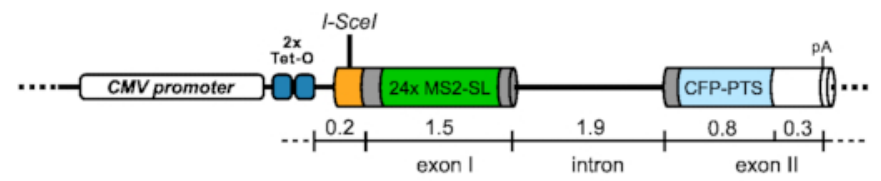

B

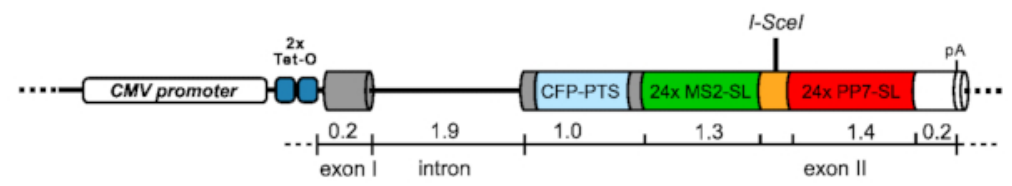

C

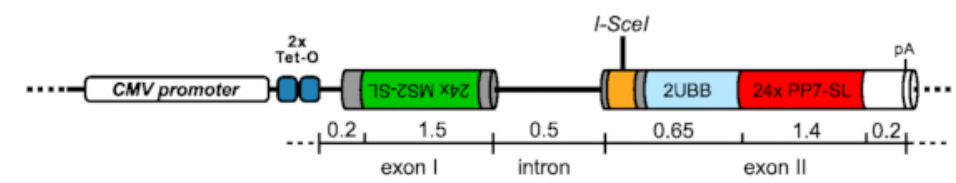

D
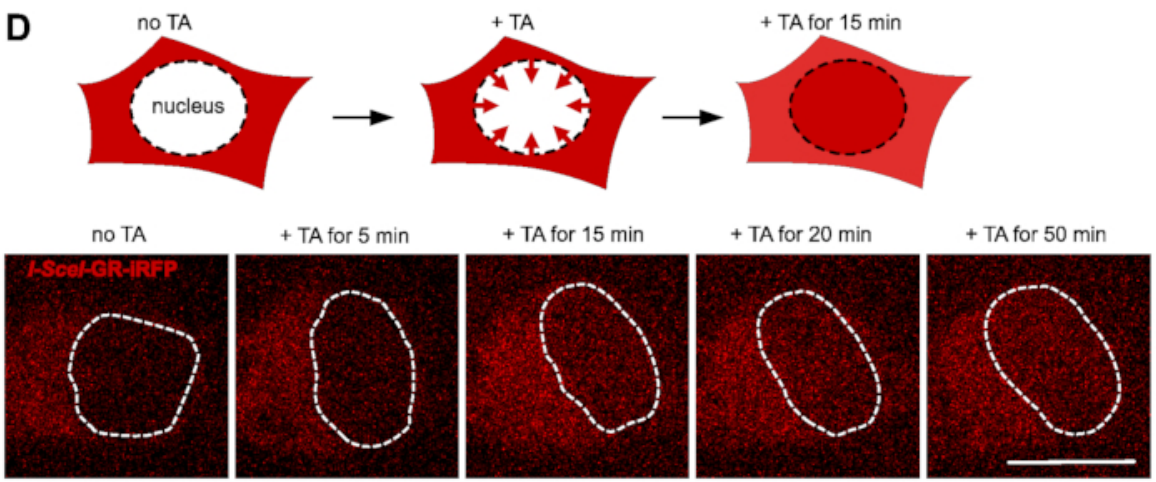

Figure 1: Reporter genes and the system to induce DNA double-strand breaks. The schematic representation in (A) to (C) shows the structure of the three reporter genes used to study transcription upon induction of a DNA double-strand break. The reporter gene with the promoter-proximal I-Scel site in the first exon (PROM) flanked downstream by a $24 x$ MS2 stemloop (MS2-SL) sequence array is depicted in (A). The reporter gene with the I-Scel site in the second exon (EX2) flanked upstream with a 24x MS2 stem-loop sequence and downstream by a 24x PP7 stem-loop (PP7-SL) array is shown in (B). The reporter gene with the I-Scel site located in exon II with an anti-parallel insertion of the 24x MS2 stem-loop sequence upstream of the I-Scel site to detect antisense transcription (EX2-AS) is shown in (C). The function of the I-Scel-GR-iRFP fusion protein construct is depicted in (D) by a graphical display and corresponding images of live cells below. Upon transient expression of the construct (red color) in the reporter gene cell lines, the protein is exclusively cytoplasmic, which prevents a premature cleavage of the target site by the I-Scel endonuclease. Upon addition of TA, the fusion protein starts migrating into the cell nucleus (indicated by a dashed line) and starts accumulating between 5-15 min. Scalebar $=10 \mu \mathrm{m}$ Please click here to view a larger version of this figure. 
A

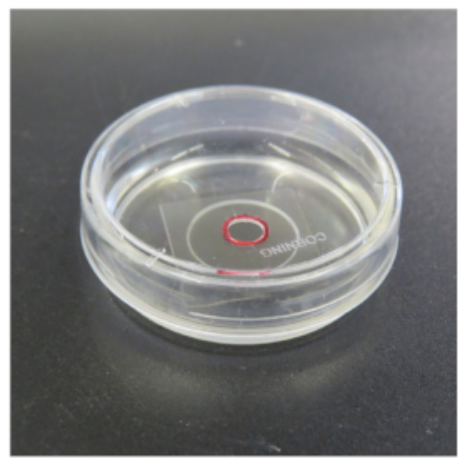

C

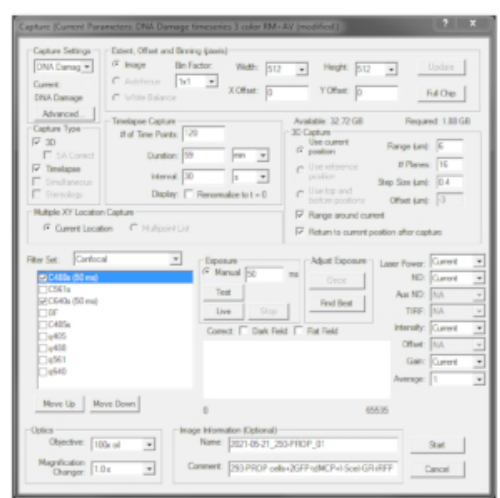

E

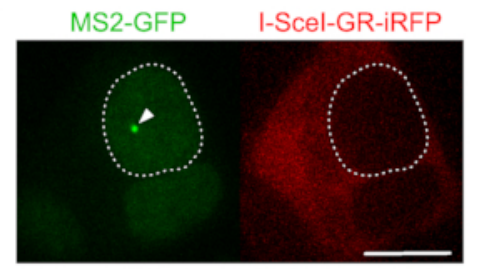

B

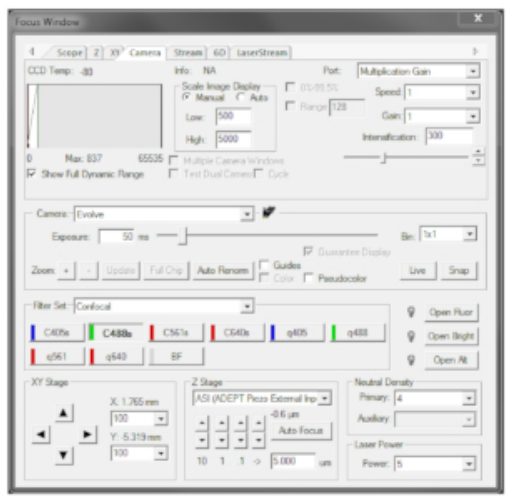

D

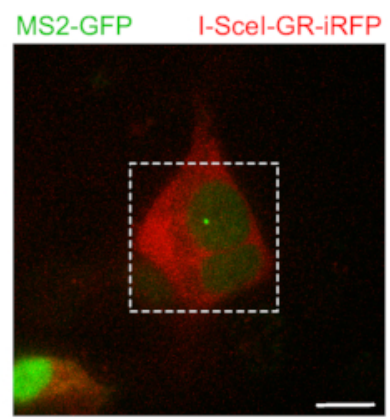

$\mathbf{F}$

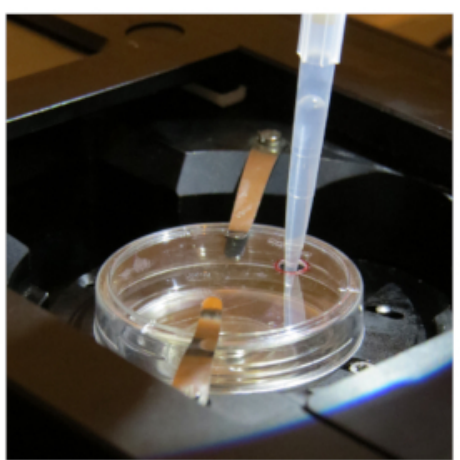

Figure 2: Selecting cells for 3D time-lapse imaging of transcription sites. The photo in (A) shows a $35 \mathrm{~mm}$ glass-bottom dish used for the live cell imaging, with a custom modified lid, in which a hole of $3 \mathrm{~mm}$ diameter was drilled to add the TA diluted in growth medium directly. The hole location is marked with a red circle. The panel in (B) shows a screenshot of the "Focus" window of the microscope control software to set up the live view exposure time, filter setting, laser intensity, and scale image display to screen for cells for subsequent time-lapse imaging in (C), the screenshot of the corresponding "Capture" window to adjust all settings for live cells' 3D time lapse acquisition. The specific settings for the Filter, Capture Type, Time-Lapse Capture, and 3D Capture panes are described in section 3. In the view shown here, the settings are adjusted to acquire a 3D time-lapse of the PROM reporter gene line transfected with the MS2-GFP and I-Scel-GR-iRFP 
constructs for imaging transcription upon induction of a DNA double-strand break in the promoter-proximal region of the reporter gene. The image in (D) is merged for the GFP and iRFP channels and shows a field of view as seen through the microscope system, with several cells of the 293-PROM reporter gene cell line. The cells were co-transfected with the tandem dimer MS2 coat protein construct fused to a nuclear localization sequence, two green fluorescent proteins (GFP-MS2CP), and the I-Scel-GR-iRFP construct. Several cells show expression of the GFP-MS2CP construct, thereby highlighting the nuclei and the I-Scel-GR-iRFP construct highlighting the cytoplasm. The dashed square indicates the magnified region shown in (E). For the 3D time-lapse imaging, cells are selected according to the requirements conferred in the Discussion, such as the cell with the larger nucleus in the magnified region in (D). This cell is transfected with both fluorescent constructs and shows a brightly labeled transcription site (arrowhead) by accumulating the GFP-MS2CP on de novo transcribed reporter gene pre-mRNAs (left image). The growth medium of the cells does not contain TA; therefore, the I-Scel-GR-iRFP construct is exclusively cytoplasmic (right image). In $(\mathbf{F})$, the glass bottom dish is mounted in the microscope stage incubation chamber for 3D time-lapse imaging experiments and equipped with the custom lid containing the TA loading hole. The $200 \mu \mathrm{L}$ micropipette tip is carefully inserted into the hole to apply the diluted TA to the growth medium of the cells. Scalebar $=10 \mu \mathrm{m}$ Please click here to view a larger version of this figure. 
A

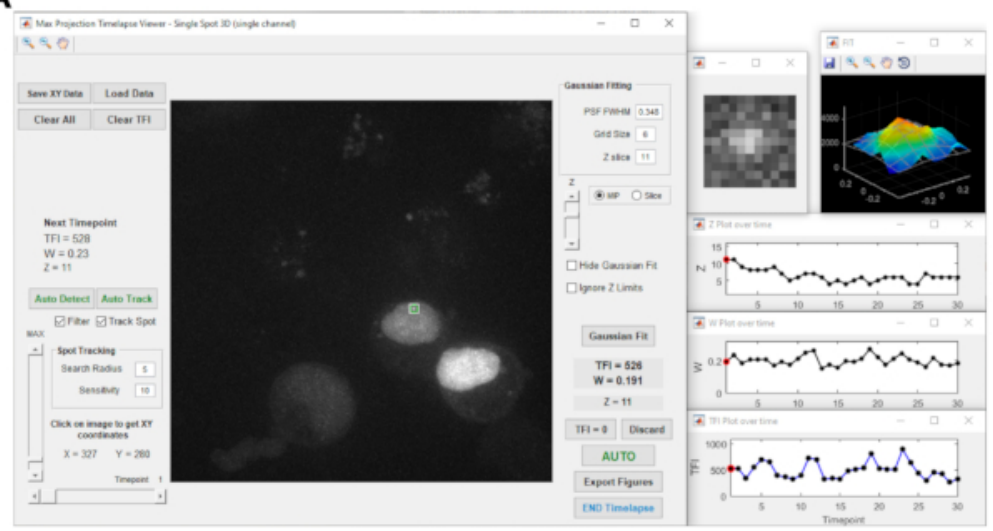

B

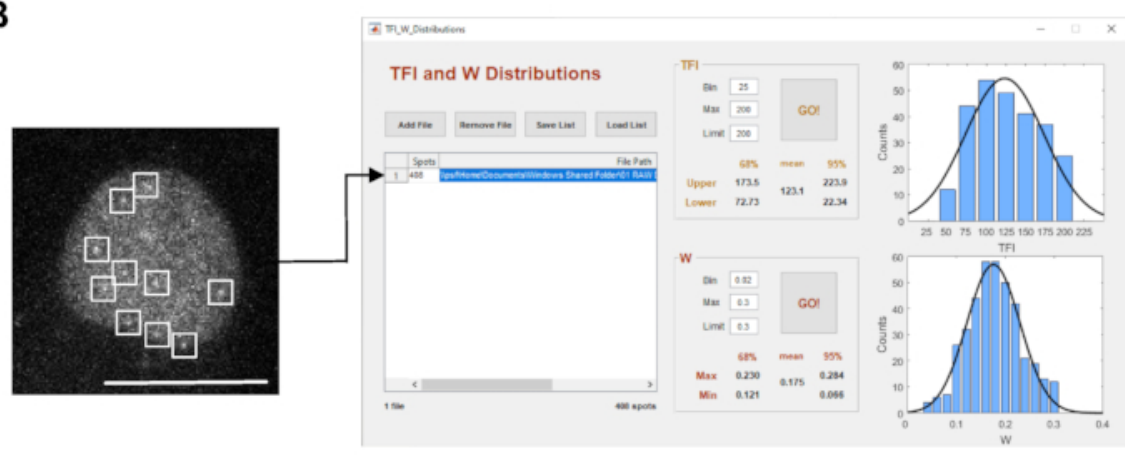

Figure 3: Image acquisition and analysis software. The panel in (A) shows a screenshot of the STaQTool: Spot tracking and quantification tool. The image displays an example time-series of the PROM reporter gene cell line with labeled transcription site marked with a green circle/white square in the maximum intensity projection display in the center. The windows on the right-side display a magnified view of the selected transcription site spot, the corresponding 3D shaded intensity surface plot with the 2D Gaussian fit grid for the current time point as well as plots of the transcription site spot $Z$ position within the z-stack, the Gaussian fit width (W) and the TFI measurement over time. The microscopic image in (B) shows a zoomed single optical plane of a nucleus of a PROM reporter gene cell line transfected with MS2-GFP. The image represents a one-time point of a 2D calibration time series with 120 timepoints. The nucleus shows several fluorescently labeled transcripts appearing as diffraction-limited objects in the nucleoplasm (marked by white squares). The right-hand side panel shows a screenshot of the TFI and W Distributions tool in the STaQTool to analyze multiple spots in 2D time-lapse acquisitions. In this example analysis, the tool detected 408 diffraction-limited spots representing reporter gene transcripts labeled with MS2-GFP that diffuse in the nucleoplasm. The graphs on the right display the TFI and Gaussian fit width distribution histograms of the objects and the Gaussian fit curves. The TFI and W mean values derived from the position of the center peak of the Gaussian fit curve and the calculated confidence intervals are displayed in the respective pane. Scalebar $=10 \mu \mathrm{m}$ Please click here to view a larger version of this figure. 
A

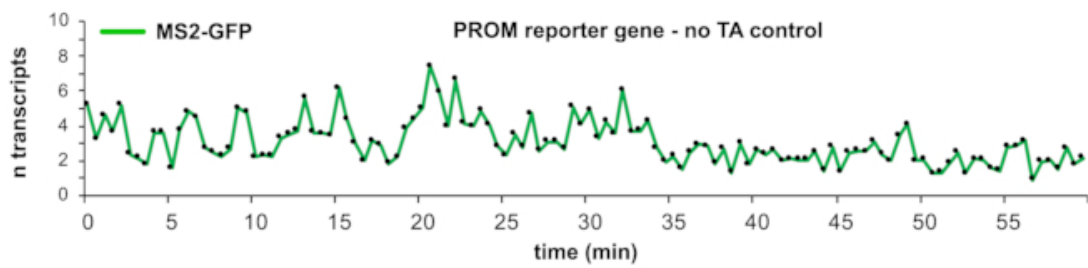

B

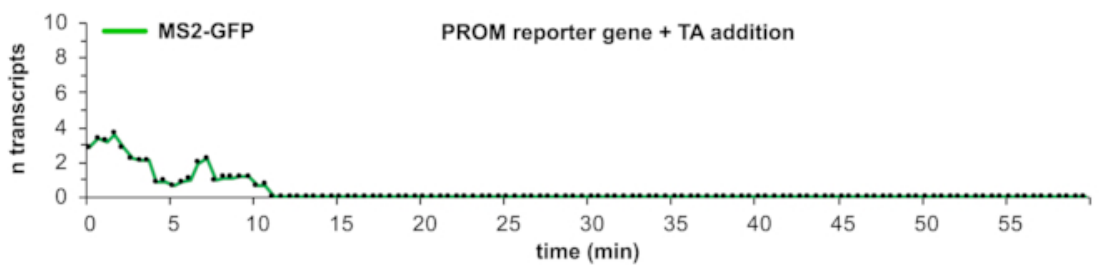

C

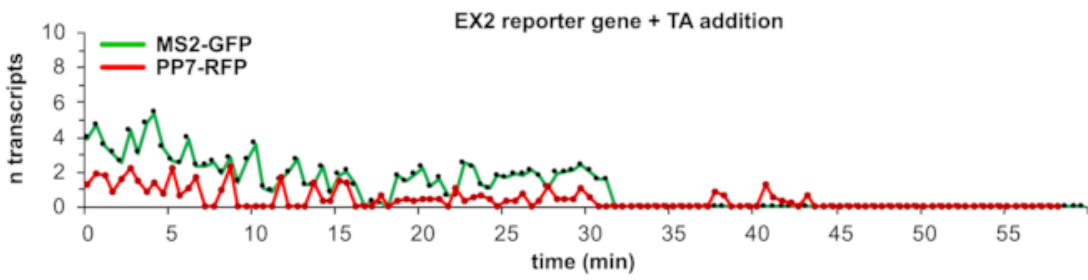

D

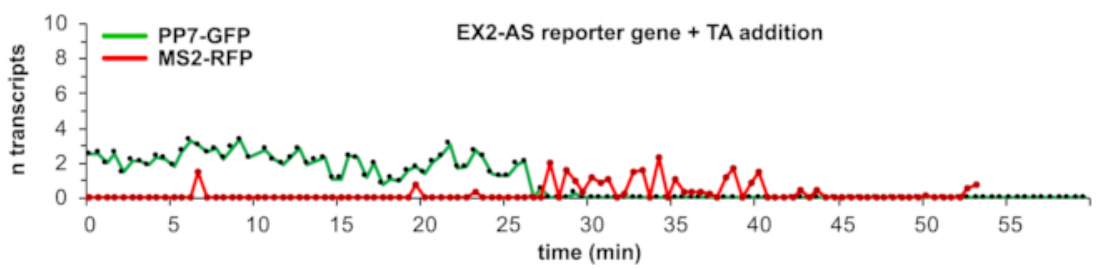

Figure 4: Representative results of transcription detection at sites of DNA double-strand breaks. The graphs in (A) to (D) represent the calibrated TFI curve of one transcription site over time. The TFI values are converted to transcripts using the mean TFI of single transcripts measured in the calibration experiments for the respective reporter gene construct and fluorescently tagged RNA stem-loop binding protein MS2 or PP7 used in the respective experiment. In (A), a graph from a control experiment using the PROM reporter gene without TA addition is shown. Transcripts labeled with MS2-GFP indicate continuous transcriptional activity over the entire observation period. The graph in (B) represents the PROM reporter gene upon TA addition and induction of a DSB which leads to a suppression of transcription for the remaining observation time. The EX2 reporter gene graph in (C) shows a transcription suppression of canonical promoter-driven transcription upon TA addition. Later in the same time-lapse, only the PP7-RFP labeled transcriptional activity emerges. In the graph in (D), the EX2-AS reporter gene transcription from the canonical promoter in sense direction is similarly suppressed upon TA addition to the EX2 reporter gene in (C). However, the appearance of MS2-RFP labeled transcripts originating from the inverse 
inserted MS2 stem-loop sequence indicates antisense transcription absent during sense transcription activity before TA addition. Please click here to view a larger version of this figure.

\begin{tabular}{|l|c|c|}
\hline Reporter cell line & EX2 and EX2-AS & PROM \\
\hline \multirow{2}{*}{ Plasmids to transfect } & $1.5 \mu \mathrm{g} \mathrm{pl-Scel-GR-iRFP}$ & $1.5 \mu \mathrm{g}$ pl-Scel-GR-iRFP \\
\cline { 2 - 3 } & $\begin{array}{r}0.65 \mu \mathrm{g} \text { pUBC-mCherry- } \\
\text { nls-tdPP7-mCherry }\end{array}$ & $0.5 \mu \mathrm{g}$ pUBC-GFP-nls-tdMS2-GFP \\
\cline { 2 - 3 } & $0.35 \mu \mathrm{g}$ pUBC-GFP-nls-tdMS2-GFP & \\
\cline { 2 - 3 } & & \\
\hline
\end{tabular}

Table 1: Transfection of the reporter gene cell lines. The table describes the transfection schemes and amounts of the different plasmids used to transfect the different reporter gene cell lines transiently.

\section{Discussion}

Conflicts between essential biological processes such as replication, transcription, DNA damage, and DNA repair have been identified as a critical source of genome instability 22 . These studies also have led to the discovery of transcription at sites of DNA damage and attributed a functional role to the break-induced transcripts in regulating DNA damage repair processes ${ }^{23}$. The new tools and the protocol described here allow further investigation of RNA Pol II transcription dynamics at DSBs. A critical point in this protocol is the generation of cell lines that contain a single copy of the reporter gene integrated into the genome. This key feature eliminates the noise created by the transcription of several reporter genes integrated with multiple copies within a single genomic locus and allows the collection of kinetic parameters of transcription dynamics and individual RNA transcripts. A crucial technical requirement to observe transcription of single reporter gene integrations is the availability of a microscope system that allows the detection of single RNA transcripts labeled with the MS2 or PP7 system in live cells ${ }^{4,12}$. Here, live-cell microscopy is performed on a Confocal Spinning Disk system mounted on an inverted microscope, equipped with $100 \mathrm{~mW}$ solid-state Lasers coupled to an acoustic-optic tunable filter as described elsewhere ${ }^{24}$. Furthermore, to study transcription at a single DSB using the reporters, individual cells must be carefully monitored to achieve the highest time resolution, which requires imaging cells for several hours, making this a low throughput assay. Still, we observe several cells in parallel with the positioning controlled by a piezo-driven microscope stage. For ensuring optimal environmental conditions for live cell observation over hours, the microscope body, including the sample stage, is placed inside a plexiglass environmental chamber. In addition, a closed stage incubation chamber is mounted on the microscope stage and connected to $\mathrm{CO}_{2}$ and humidity supply controllers.

The first critical step in the protocol is the selection of regions of interest with cells for imaging. Each $X Y$ position marked for imaging must contain one or more cells showing transfection with the fluorescent RNA stem-loop binding proteins according to the reporter gene and transfection scheme described in Section 1, Table 1, as well as in Figure 2D, E. Furthermore, the cells must exhibit bright labeled transcription sites must be co-transfected with the I-Scel-GR- 
iRFP713 construct, and the protein must be localized initially in the cytoplasm (Figure 2D and E).

The cells should show a fluorescence intensity level of unbound fluorescently tagged MS2 and/or PP7 coat protein low enough to detect single labeled transcripts over the background fluorescence level. At the same time, a robust fluorescence intensity level of the fluorescently tagged MS2 and/or PP7 coat proteins is necessary to allow imaging over at least 60 min without losing too much fluorescence due to some bleaching that occurs. The "Scale image display" with a fixed range as described in Section 3.7 is used to allow a standardized selection of cells according to their fluorescence intensity level.

A second critical protocol step is adding the TA to the cells on pre-determined $X Y$ positions through the small hole in the lid of the glass bottom dish. Any manipulation of the glass bottom dish would cause a shift from the marked $X Y$ position of the cells and must be avoided. Therefore, carefully handling the micropipette while adding the TA diluted in the cellular growth medium is vital for pre-selected cells' successful observation, as demonstrated in Figure 2F. The adaptation of different systems to add drugs to cells mounted on a microscope stage, such as a perfusion system, would require a separate stage incubation chamber with tube entrance and exit openings and a pump or injection system to administer drugs. Other methods such as channel slides with coversliplike bottom surfaces result in a slow diffusion of administered drugs into the channel and cause an additional delay between drug addition and effect. Finally, incautious pipetting into a channel slide opening may shift the sample position as well. Therefore, the present system with a custom drilled hole in the lid of a glass-bottom dish is straightforward to adapt, low cost, and suitable for administering different growth media, drugs, and components. The small diameter of the hole and the humidified atmosphere in the stage incubation chamber also prevents drying out of the cell medium.

A third critical step in this protocol is the data analysis, which requires a manual inspection of the time points when transcription ceases due to the induction of a DSB. The time point of terminating transcription is indicated by releasing the last transcripts from the previously bright labeled site of the reporter gene transcription. Similarly, the events of breakinduced transcription initiation must be inspected with care to detect individual transcription events with the relatively low signal-to-noise ratio of single fluorescently labeled mRNAs.

The dynamics of repair of the induced DSB adds an extra layer of complexity to the analyses of data generated using these reporters, limiting them to the first minutes immediately after induction of the DSB. The transgenic nature of reporter genes and the repeat-rich nature of the MS2 and PP7 stemloop arrays may assemble a unique chromatin landscape, interfering with establishing putative stable break-induced transcription programs. Nonetheless, compared to ionizingor UV-irradiation, the I-Scel mediated induction of a DSB in reporter genes is a much more robust system to investigate transcription at individual DSBs.

Different endonuclease systems such as I-Crel, I-Ppol, or AsiS/ that have or don't have additional recognition sites within the human genome can be combined with the present reporter gene systems for a possible higher efficiency of generating DSBs. However, they require first introducing the endonuclease recognition site into the reporter genes. Secondly, they may have a similar variability on the timing and efficiency induction of a DSB in individual cells. On the other hand, inserting tandem copies of endonuclease recognition sites may increase the efficiency of DSB induction. Moreover, 
testing the presented reporter gene systems in different cell lines would allow the comparison of transcription dynamics at DSB sites between different cellular backgrounds and the availability of different DNA damage repair pathways such as in cancer cells, primary cells, and differentiated non-cycling cells. However, the construction of the reporter genes to be compatible with the Flp/FRT system is currently limiting the integration into available Flp/FRT host cell lines.

In addition to microscopy-based applications, the current reporter genes may also be combined with biochemical assays, such as chromatin immunoprecipitation, to study the recruitment of DNA repair or transcription factors to a single DSB or to assess nucleosome occupancy, histone modifications, and chromatin state around the DSB site. Furthermore, a combination with different reporter systems would allow the study of functional links between DNA damage and processes like genome organization or DNA replication.

\section{Disclosures}

The authors have no conflict of interests to disclose.

\section{Acknowledgments}

We thank RH Singer, J.A. Chao, T. Misteli, M. CarmoFonseca for gifts of plasmids and reagents. We are also indebted to the iMM Bioimaging Facility staff, A. Temudo, A. Nascimento, and J. Rino, for critical reading of the manuscript. This work was funded by PTDC/MEDOUT/32271/2017, PTDC/BIA-MOL/30438/2017 and PTDC/ MED-OUT/4301/2020 from Fundação para a Ciência e a Tecnologia (FCT), Portugal and by LISBOA-01-0145FEDER-007391, project cofunded by FEDER through POR Lisboa, Portugal 2020-Programa Operacional Regional de Lisboa, and FCT. Funding was also received from EU Horizon
2020 Research and Innovation Programme (RiboMed 857119). M.A. is the recipient of the FCT Ph.D. fellowship 2020.05899.BD.

\section{References}

1. Jackson, S.P.; Bartek, J. The DNA-damage response in human biology and disease. Nature. 461 (7267), 1071-1078 (2009).

2. Capozzo, I.; Iannelli, F.; Francia, S.; d'Adda di Fagagna, F. Express or repress? The transcriptional dilemma of damaged chromatin. FEBS Journal. 284 (14), 2133-2147 (2017).

3. Michelini, F. et al. Damage-induced IncRNAs control the DNA damage response through interaction with DDRNAs at individual double-strand breaks. Nature Cell Biology. 19 (12), 1400-1411 (2017).

4. Vítor, A. C. et al. Single-molecule imaging of transcription at damaged chromatin. Science Advances. 5 (1), eaau1249 (2019).

5. Michalik, K. M.; Böttcher, R.; Förstemann, K. A. Small RNA response at DNA ends in Drosophila. Nucleic Acids Research. 40 (19), 9596-9603 (2012).

6. Wei, W. et al. A role for small RNAs in DNA double-strand break repair. Cell. 149 (1), 101-112 (2012).

7. Francia, S. et al. Site-specific DICER and DROSHA RNA products control the DNA-damage response. Nature. 488 (7410), 231-235 (2012).

8. Vítor, A. C.; Huertas, P.; Legube, G.; de Almeida, S. F. Studying DNA double-strand break repair: An evergrowing toolbox. Frontiers in Molecular Biosciences. 7, $24(2020)$. 
9. Alt, F. W. et al. Synthesis of secreted and membranebound immunoglobulin mu heavy chains is directed by mRNAs that differ at their 3' ends. Cell. 20 (2), 293-301 (1980).

10. Watakabe, A.; Tanaka, K.; Shimura, Y. The role of exon sequences in splice site selection. Genes \& Development. 7 (3), 407-18 (1993).

11. Guth, S.; Martínez, C.; Gaur, R. K.; Valcárcel, J. Evidence for substrate-specific requirement of the splicing factor $\operatorname{UAF}(35)$ and for its function after polypyrimidine tract recognition by $\operatorname{U} 2 \mathrm{AF}(65)$. Molecular and Cellular Biology. 19 (12), 8263-71 (1999).

12. Martin, R. M.; Rino, J.; Carvalho, C.; Kirchhausen, T.; Carmo-Fonseca, M. Live-cell visualization of pre-mRNA splicing with single-molecule sensitivity. Cell Reports. 4 (6), 1144-55 (2013).

13. Peabody, D.S. The RNA binding site of bacteriophage MS2 coat protein. The EMBO Journal. 12 (2), 595-600 (1993).

14. Lim, F.; Peabody, D. S. RNA recognition site of PP7 coat protein. Nucleic Acids Research. 30 (19), 4138-44 (2002).

15. Chao, J. A.; Patskovsky, Y.; Almo, S. C.; Singer, R. H. Structural basis for the coevolution of a viral RNA-protein complex. Nature Structural \& Molecular Biology. 15 (1), 103-5 (2008).

16. Bertrand, E.; Chartrand, P.; Schaefer, M.; Shenoy, S. M.; Singer, R. H.; Long, R. M. Localization of ASH1 mRNA particles in living yeast. Molecular Cell. 2 (4), 437-445 (1998).

17. Larson, D. R.; Zenklusen, D.; Wu, B.; Chao, J. A.; Singer, R. H. Real-time observation of transcription initiation and elongation on an endogenous yeast gene. Science. 332 (6028), 475-478 (2011).

18. Soutoglou, E. et al. Positional stability of single doublestrand breaks in mammalian cells. Nature Cell Biology. 9 (6), 675-682 (2007).

19. Rouet, P.; Smih, F.; Jasin, M. Introduction of doublestrand breaks into the genome of mouse cells by expression of a rare-cutting endonuclease. Molecular and Cellular Biology. 14 (12), 8096 (1994).

20. Roukos, V.; Voss, T. C.; Schmidt, C. K.; Lee, S.; Wangsa, D.; Misteli, T. Spatial Dynamics of Chromosome Translocations in Living Cells. Science (New York, N.Y.). 341 (6146), 660 (2013).

21. Rino, J.; de Jesus, A. C.; Carmo-Fonseca, M. STaQTool: Spot tracking and quantification tool for monitoring splicing of single pre-mRNA molecules in living cells. Methods. 98, 143-149 (2016).

22. Hamperl, S.; Bocek, M. J.; Saldivar, J. C.; Swigut, T.; Cimprich, K. A. Transcription-replication conflict orientation modulates R-Loop levels and activates distinct DNA damage responses. Cell. 170 (4), 774-786.e19 (2017).

23. D'Alessandro, G.; d'Adda di Fagagna, F. Transcription and DNA Damage: Holding Hands or Crossing Swords? Journal of Molecular Biology. 429 (21), 3215-3229 (2017).

24. Boulant, S.; Kural, C.; Zeeh, J. C.; Ubelmann, F.; Kirchhausen, T. Actin dynamics counteract membrane tension during clathrin-mediated endocytosis. Nature Cell Biology. 13 (9), 1124-1131 (2011). 